\title{
Glassy features of crystal plasticity
}

\author{
Arttu Lehtinen, ${ }^{1}$ Giulio Costantini, ${ }^{2}$ Mikko J. Alava, ${ }^{1}$ Stefano Zapperi,,${ }^{1,2,3,4}$ and Lasse Laurson ${ }^{1}$ \\ ${ }^{1}$ COMP Centre of Excellence, Department of Applied Physics, Aalto University, P.O. Box 11100, FI-00076 Aalto, Espoo, Finland \\ ${ }^{2}$ Center for Complexity and Biosystems, Department of Physics, University of Milano, via Celoria 26, 20133 Milano, Italy \\ ${ }^{3}$ ISI Foundation, Via Alassio 11/C, 10126 Torino, Italy \\ ${ }^{4}$ CNR-IENI, Via R. Cozzi 53, 20125 Milano, Italy
}

(Received 10 November 2015; revised manuscript received 21 July 2016; published 2 August 2016)

\begin{abstract}
Crystal plasticity occurs by deformation bursts due to the avalanchelike motion of dislocations. Here we perform extensive numerical simulations of a three-dimensional dislocation dynamics model under quasistatic stress-controlled loading. Our results show that avalanches are power-law distributed and display peculiar stress and sample size dependence: The average avalanche size grows exponentially with the applied stress, and the amount of slip increases with the system size. These results suggest that intermittent deformation processes in crystalline materials exhibit an extended critical-like phase in analogy to glassy systems instead of originating from a nonequilibrium phase transition critical point.
\end{abstract}

DOI: 10.1103/PhysRevB.94.064101

\section{INTRODUCTION}

Plastic deformation of crystalline solids, mediated by the stress-driven motion of crystal dislocations, has been shown to be a highly heterogeneous and wildly fluctuating process [1-3], in analogy to numerous other driven systems exhibiting "crackling noise" [4]. Broad, power-law-like distributions of strain bursts are observed in experiments on micron-scale samples [5-13], and the same is often true for acoustic emission (AE) amplitudes in the case of larger specimens [14-16]. While the bursty nature of crystal plasticity is a well established fact, the question of its nature and origin remains a subject of lively debate $[8,9,17]$.

To address such questions in an appropriate fashion, high quality numerical studies of realistic discrete dislocation dynamics (DDD) models, capturing the avalanchelike deformation process, are essential [7,17-19]. The majority of DDD studies of dislocation avalanches have so far been performed using relatively simple and computationally efficient $2 \mathrm{D}$ systems, describing pointlike cross sections of ensembles of straight, parallel edge dislocations [17-19]. Real threedimensional plastically deforming crystals are not described in all their aspects by the 2D DDD models [20]. In 3D, dislocations are flexible lines (exhibiting in general a mixture of edge and screw character) gliding along multiple slip planes and interacting in addition to the long-range elastic stress fields also via various short-range dislocation reactions [21] (junction formation, annihilation, etc.). During the deformation process dislocation density typically increases due to, e.g., growth of dislocation loops and via the activation of Frank-Read sources, thus leading to strain hardening of the material. It is tempting to attribute the complexity to an underlying phase transition with divergent correlations, so that for high stresses above the yield stress continuous flow would ensue. A scaling picture related to mean-field-like behavior (due to long-range interactions) and a pinning/depinning transition (arising from the mutual interactions among moving and jammed dislocations), has been proposed [7-9]. However, dislocations do not in general move in the presence of a static pinning field, and therefore they tend to "jam" instead of getting pinned; moreover, their mutual interactions are anisotropic and nonconvex, implying that, e.g., the no-passing theorem would not be applicable.
In this paper, we present results from an extensive study of dislocation avalanches in a fully 3D DDD model. We show that the bursty 3D plastic deformation process exhibits scale-free features already at the beginning of the stress-strain curve. The small-scale and large-scale ("collective") avalanches have different scaling exponents, and the average avalanche size increases exponentially with the applied stress, in analogy with the dynamics observed in simple 2D models [17,18] which, however, miss completely the strain hardening present in 3D. In our DDD simulations of Al single crystals, performed using the ParaDis [22] code, we employ a quasistatic stresscontrolled loading protocol. This eliminates possible rate effects in the avalanche statistics such as occur, e.g., in the ABBM model of mean-field avalanches [23]. Our detailed statistical analysis of the sizes (for durations see Supplemental Material [24]) of the deformation bursts-encompassing both stress-resolved and integrated probability distributionsreveals a novel scaling picture which is at odds with the meanfield depinning scenario [1,7-9]. Instead, plasticity of FCC single crystals is found to exhibit an extended, critical-like phase, with the amount of slip within strain bursts diverging with the system size at any applied stress, hinting at a systemspanning correlation length. We attribute such behavior to the glassy properties of the dislocation system $[17,25]$, originating from frustrated dislocation interactions.

\section{SIMULATIONS}

In this paper we use a modified version of the DDD code ParaDis [22]. In ParaDis dislocations are modeled using a nodal discretization scheme: Dislocation lines are represented by nodal points connected to their neighbors by straight segments. Changes in dislocation geometry are made possible by adding and removing these nodal points. The total stress acting on a node consists of the external part, resulting from the deformation of the whole crystal, and of the internal, anisotropic stress fields generated by the other dislocations within the crystal. The latter stress fields are computed by applying the well-known results of linear elasticity theory to the straight segments between nodes. Both of these fields generate forces which move the discretization nodes. The 
external stress generates a Peach-Koehler force which is applied to all nodes. The forces between dislocations themselves are divided to local and far field ones. Forces between segments of nearby nodes and self-interaction of dislocations are calculated with explicit line integrals. Far-field forces are calculated from the coarse-grained dislocation structure using a multipole expansion. Near the dislocation core, local interactions, such as junction formation, annihilation, etc., are introduced phenomenologically with input from smaller scale simulation methods (e.g., MD) and experimental results. Once the forces are known, a trapezoidal integrator is used to solve the equation of motion for the discretization nodes. However it must be taken into account that in real materials, the motion of dislocations is subject to constraints which depend on the underlying crystal structure (e.g., FCC or $\mathrm{BCC}$ ) and the nature of the dislocations (e.g., screw or edge) in a complicated manner. These details are encoded in the material-specific mobility function which relates the total forces experienced by dislocations to their velocities. In order to simulate bulk properties we use periodic boundary conditions (PBC). These are implemented by using an Ewalds sum procedure similar to those used in atomistic simulations with periodic structures and long-range interactions. The main simulation cell is surrounded by periodic image cells which contain the images of the segments in the main cell. Interaction stresses between a given segment and its images are obtained from precomputed tables which contain the possible image stresses from differential segments as a function of dislocation orientation and Burgers vector [26].

We consider here the FCC crystal structure with material parameters of $\mathrm{Al}$ (shear modulus $G=26 \mathrm{GPa}$, Poisson ratio 0.35 , Young modulus $70.2 \mathrm{GPa}$, Burgers vector $b=$ $2.863 \times 10^{-10} \mathrm{~m}$, and dislocation mobility $10^{4} \mathrm{~Pa}^{-1} \mathrm{~s}^{-1}$; for simplicity, both edge and screw segments are taken to have the same mobility) and employ periodic boundary conditions. The typical maximum strains in the simulations are of the order of $1 \%$, limited by computational cost and what is physically feasible given the boundary conditions. To study the effect of the system size, we consider different linear sizes $L=0.715,1.001,1.2298,1.43$, and $2.1473 \mu \mathrm{m}$ of the cubic simulation box (i.e. within the range of those of typical microcrystal compression experiments [5,6,8-12]), keeping the initial dislocation density roughly constant at $\rho_{0} \approx 3.0 \times 10^{13} 1 / \mathrm{m}^{2}$; this leads to initial numbers $N_{0}=10$, $20,30,40$, and 80 of straight mixed dislocations placed randomly on the glide planes of the FCC lattice. Results are shown in scaled units, by measuring lengths, stresses, strains, and times in units of $\rho_{0}^{-1 / 2}, G b \rho_{0}^{1 / 2}, b \rho_{0}^{1 / 2}$, and $\left(G b^{2} M \rho_{0}\right)^{-1}$, respectively.

The random initial configurations are first relaxed in zero applied stress, a process during which the dislocation network evolves towards a (meta)stable state where the initially straight dislocation lines exhibit some curvature. After relaxation, the quasistatic stress-controlled driving is initiated; to test the robustness of our results, we employ two different driving protocols, and also consider simulations with (Supplemental Material [24]) and without (results shown in the main paper) cross-slip; while cross-slip affects the hardening rate (Supplemental Material [24]), the strain burst statistics is unaffected by it. The dislocation activity is measured either by
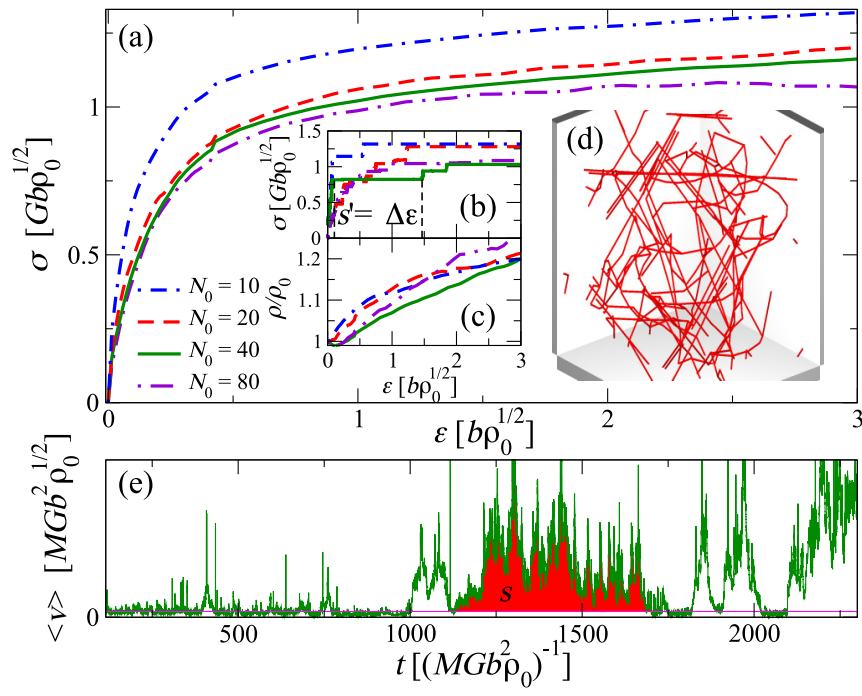

FIG. 1. (a) The average stress-strain curves $\sigma(\epsilon)$ for different system sizes $N_{0}$, revealing a size effect ("smaller is stronger"). (b) Examples of individual, staircaselike stress-strain curves, also showing the definition of a strain burst avalanche size $s^{\prime}=\Delta \epsilon$. (c) Average evolution of the dislocation density $\rho$ with $\epsilon$. (d) An example of the dislocation configuration with $N_{0}=40$, deformed up to $\epsilon=3 b \rho^{0.5}$. (e) A typical time series of the average dislocation velocity $\langle V\rangle(t)$; the red area under a burst shows an example of the definition of the size $s$ of a velocity avalanche.

the absolute collective segment-length weighted dislocation velocity $V(t)=\left(\sum_{i} l_{i} v_{\perp, i}\right) /\left(\sum_{i} l_{i}\right)$ (with $l_{i}$ and and $v_{\perp, i}$ the length and velocity perpendicular to the line direction of the $i$ th dislocation segment, respectively) or by the strain rate $\dot{\epsilon}(t)$ (originating from dislocations moving in the direction of the resolved applied shear stress). When the activity falls below a small threshold $V_{\text {th }}$, the external stress $\sigma_{\text {ext }}$ is increased at a constant rate (we consider $\dot{\sigma}_{\text {ext }}=2.5 \times 10^{13} \mathrm{~Pa} / \mathrm{s}$, or 0.0011268 in the scaled units, unless stated otherwise). When $V(t)$ [or $\dot{\epsilon}(t)$, depending on the protocol used] exceeds the threshold, $\sigma_{\mathrm{ext}}$ is kept constant until the avalanche has finished, and $V(t)[\dot{\epsilon}(t)]$ again falls below the threshold. Here, we focus on velocity avalanches defined by thresholding the $V(t)$ signal, with the avalanche size defined as $s=\int_{0}^{T}\left[V(t)-V_{\mathrm{th}}\right] \mathrm{d} t[T$ is the duration of the avalanche such that $V(t)$ continuously exceeds $\left.V_{\text {th }}\right]$; details on other protocols and avalanche definitions (e.g., $s^{\prime}$ defined as the strain increment $\Delta \epsilon$ ), leading to essentially the same results, are provided as Supplemental Material [24], along with an example animation of the bursty deformation process. Figure 1 shows examples of the simulated average and individual stress-strain curves, the evolution of the dislocation density, a snapshot of a deformed dislocation configuration, as well as an example of a $V(t)$ signal, including also an illustration of the definition of $s$.

\section{RESULTS}

The stress-integrated avalanche size distribution $P_{\mathrm{INT}}(s)$, i.e., the distribution of all avalanches irrespective of the $\sigma$ value at which they occur, is the quantity measured in many experiments $[5,6,10-12,27]$. Figure 2 shows our $P_{\mathrm{INT}}(s)$ from 


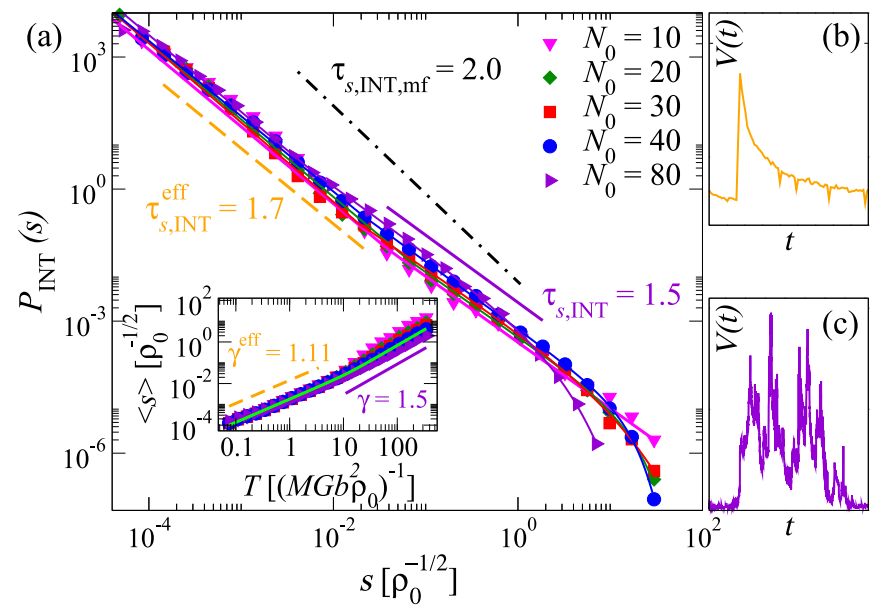

FIG. 2. (a) Main panel: stress-integrated avalanche size distributions $P_{\mathrm{INT}}(s)$ for different $N_{0}$. The data is fitted with the crossover scaling form [Eq. (1), solid lines], revealing two power law regimes, with small avalanches [an example $V(t)$ signal shown in (b)] characterized by $\tau_{s, \text { INT }}^{\text {eff }}=1.75 \pm 0.03$, while larger avalanches [an example $V(t)$ signal shown in (c)] have $\tau_{s, \mathrm{INT}}=1.52 \pm 0.04$ (for $N_{0}=80$; considering smaller $N_{0}$ yields similar results). For comparison, the dash-dotted line corresponds to the mean-field result $\tau_{s, \mathrm{INT}, \mathrm{MF}}=2$. The inset of (a) displays the $\langle s(T)\rangle$ relation, with $\gamma=1.50 \pm 0.02$ and $\gamma^{\text {eff }}=1.11 \pm 0.02$ for $T \gg T^{*}$ and $T \ll T^{*}$, respectively.

the 3D DDD simulations for different $N_{0}$; These exhibit two power law regimes, with a crossover scale $s^{*}$ separating scaling regimes of "small" and "large" avalanches; analysis of the strain burst distributions $P\left(s^{\prime}\right)$ (Supplemental Material [24]) shows that $s^{*}$ corresponds roughly to the characteristic strain burst size $s_{1}^{\prime} \propto 1 / N_{0}$, i.e., the strain accumulated due to one dislocation moving one average dislocation spacing. Thus, our data is well described by a crossover scaling form [28]

$$
P_{\mathrm{INT}}(s)=\frac{A s^{-\tau_{s, \mathrm{INT}}}}{e^{\left(\frac{s}{s_{0}}\right)^{b}}}\left[1+\left(\frac{s}{s^{*}}\right)^{\left(\tau_{s, \mathrm{INT}}-\tau_{s, \mathrm{fNT}}^{\mathrm{eff}}\right) \kappa}\right]^{\frac{1}{\kappa}},
$$

where $\kappa$ controls the sharpness of the crossover between the two power laws with exponents $\tau_{s, \mathrm{INT}}$ and $\tau_{s, \mathrm{INT}}^{\text {eff }}$, and $s_{0}$ is the cutoff avalanche size, arising here due to the maximum strain reached in the simulations. We find $\tau_{s, \mathrm{INT}}=$ $1.52 \pm 0.04$ for $s \gg s^{*}$ in the $N_{0}=80$ system (similar values are obtained for smaller $N_{0}$ ), spanning almost three orders of magnitude, while for $s \ll s^{*}$ a larger effective exponent $\tau_{s, \mathrm{INT}}^{\text {eff }}=1.75 \pm 0.03$ ensues. The latter avalanches are small and temporally asymmetric [typically consisting of a small jump of an individual dislocation, followed by relaxation, see Fig. 2(b)] like avalanches triggered by local perturbations in a 2D DDD model [18]. Experimental values are scattered around $\tau_{s, \mathrm{INT}}=1.5$, with some variation between different experiments [5,6,10-12], in good agreement with our largeavalanche regime [see also Fig. 2(c)]; notice that due to limited resolution, the small-avalanche regime is not accessible in typical experiments.

In the inset of Fig. 2(a), we show the scaling of the average avalanche size $\langle s(T)\rangle$ with the avalanche duration $T$; again two scaling regimes can be observed, and fitting a crossover scaling

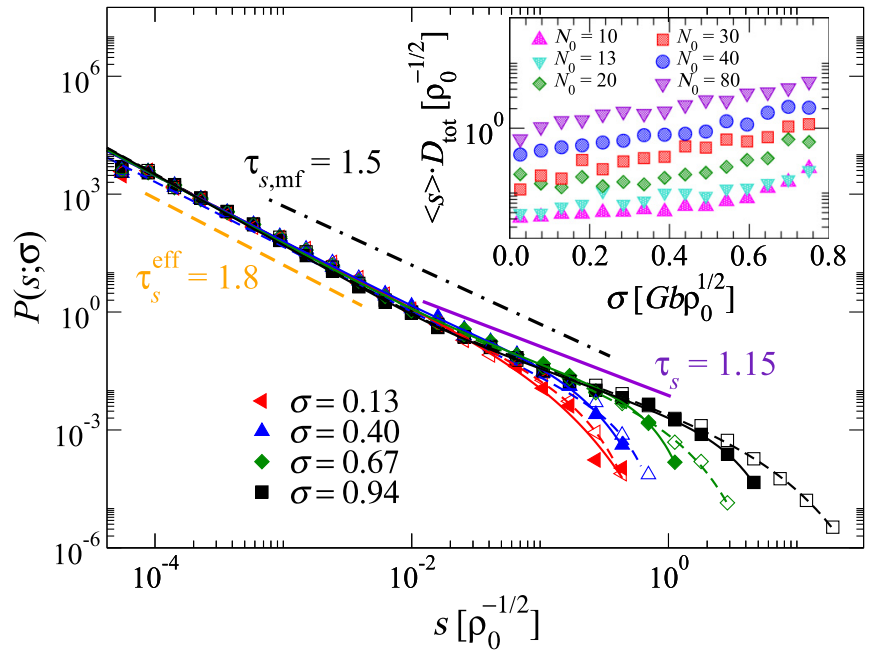

FIG. 3. Stress-resolved avalanche size distributions $P(s, \sigma)$ for different stress levels $\sigma$ (main figure; open and filled symbols correspond to $N_{0}=40$ and 80 , respectively). The data is fitted with the crossover scaling form of Eq. (1) (solid lines), revealing $\tau_{s}=1.18 \pm 0.06$ for $s \gg s^{*}$, and a larger effective $\tau_{s}^{\text {eff }}=1.80 \pm 0.04$ for $s \ll s^{*}$ (for $N_{0}=80$; smaller $N_{0}$ 's yield similar values). The inset shows the average avalanche size $\langle s\rangle D_{\text {tot }}$ as a function of $\sigma$ for various $N_{0}$, revealing a roughly exponential $\sigma$ dependence, and an increasing average avalanche size with $N_{0}$ at a fixed $\sigma$.

form $\langle s(T)\rangle=B T^{\gamma}\left[1+\left(T / T^{*}\right)^{\left(\gamma^{\text {eff }}-\gamma\right) \kappa}\right]^{1 / \kappa}$ to the $N_{0}=40$ data results in $\gamma^{\text {eff }}=1.11 \pm 0.02$ for $T \ll T^{*} \approx 12$, and $\gamma=1.50 \pm 0.02$ for $T \gg T^{*}$; the latter may be contrasted with the mean-field depinning value $\gamma_{\mathrm{MF}}=2$. The large avalanche regime has a system size dependent prefactor which can be scaled away by considering an alternative, "extensive" measure of the avalanche size, e.g., the accumulated slip $\langle d\rangle \equiv\langle s\rangle L^{2}$ (Supplemental Material [24]).

It has been proposed that observations of $\tau_{s, \text { INT }} \approx 1.5$ may be compatible with mean-field depinning if, due to back stresses induced by strain hardening, the system is constantly pushed towards a critical yield stress, in the spirit of self-organized criticality (SOC) $[7,29,30]$, resulting in a stationary avalanche process. In Fig. 3, we consider the stress-resolved avalanche size distributions, i.e., $P(s ; \sigma)$ of avalanches within stress bins centered at $\sigma$, as also reported for some experiments $[8,9]$. Fitting the scaling form of Eq. (1) to the $P(s ; \sigma)$ distributions [with substitutions $P_{\mathrm{INT}}(s) \rightarrow$ $P(s ; \sigma), \tau_{s, \mathrm{INT}} \rightarrow \tau_{s}$, and $\left.\tau_{s, \mathrm{INT}}^{\text {eff }} \rightarrow \tau_{s}^{\text {eff }}\right]$ reveals a largeavalanche exponent $\tau_{s}<\tau_{s, \mathrm{INT}}$, a signature of nonstationary avalanche processes [31]; for the largest stress bin in Fig. 3, we obtain $\tau_{s}=1.18 \pm 0.06$, while for $s \ll s^{*}$, a larger effective $\tau_{s}^{\text {eff }}=1.80 \pm 0.04$ is again observed $\left(N_{0}=80 ;\right.$ smaller $N_{0}$ 's yield similar values). The avalanche cutoff scale $s_{0}(\sigma)$ grows with the stress level $\sigma$. This, together with the fact that the $\tau_{s}$ exponent is significantly smaller than the mean-field [8] or ABBM [23] value of 1.5 (notice that we have eliminated possible rate effects by employing the quasistatic driving protocol, and also verified the independence of the results on the stress rate, see Supplemental Material [24]), provides strong evidence suggesting that our avalanches cannot be described by mean-field depinning. The same values for $\tau_{s}$ 


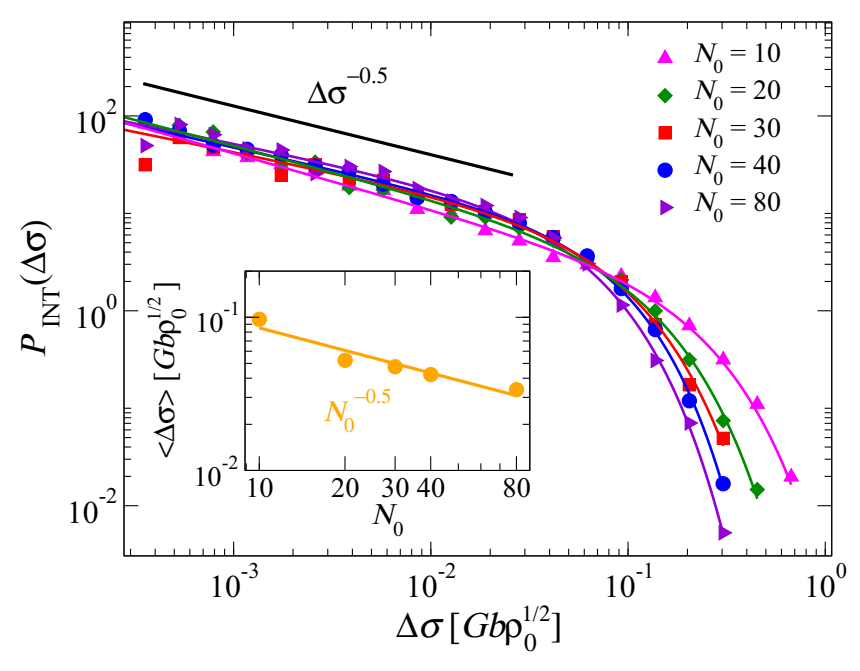

FIG. 4. Main figure shows the strain-integrated stress increment distributions $P_{\mathrm{INT}}(\Delta \sigma)$ for different $N_{0}$, revealing a cutoff decreasing with the system size. The inset shows that the average stress jump magnitude $\langle\Delta \sigma\rangle$ decreases as $N_{0}^{-0.5}$.

and $\tau_{s, \text { INT }}$ can be extracted also from the complementary cumulative distributions functions (CDFs, see Supplemental Material [24]), highlighting the robustness of the values. We also note that these, together with the exponents of the duration distributions $\left(\tau_{T}=1.22 \pm 0.14\right.$ and $\tau_{T}^{\text {eff }}=1.92 \pm$ 0.08 , Supplemental Material [24]) fulfill the scaling relations $\gamma=\left(\tau_{T}-1\right) /\left(\tau_{s}-1\right)$ and $\gamma^{\text {eff }}=\left(\tau_{T}^{\text {eff }}-1\right) /\left(\tau_{s}^{\text {eff }}-1\right)$ within error bars above and below the crossover, respectively. The values of $\tau_{s}^{\text {eff }}, \tau_{T}^{\text {eff }}$, and $\gamma^{\text {eff }}$ are close to those found recently for avalanches triggered by local perturbations in a 2D DDD model [18]. Furthermore, our results are not sensitive to details of the preparation of the initial state, as evidenced by considering systems with a loading history as initial states (Supplemental Material [24]).

To further characterize the stress dependence of the avalanche sizes, we show the scaling of the average total dislocation activity $\langle s\rangle D_{\text {tot }}$ vs $\sigma$ (with $s$ computed from the average velocity, and $D_{\text {tot }}=\sum_{i} l_{i}$ the total dislocation line length of the system) in the inset of Fig. 3. We observe that the avalanche size increases roughly exponentially with stress for all system sizes $N_{0}$, and at any given stress it depends significantly on $N_{0}$. This is in contrast to a standard depinning transition where the avalanche size is independent on $N_{0}$ unless the stress is close to the depinning point. Similar results are, however, obtained in simplified 2D DDD models $[17,18]$ and experiments [10]. Our results indicate that rather than the applied stress, the limiting factor for the amount of dislocation activity within the strain bursts is the finite system size [32]. Thus, the system appears to exhibit an extended, critical-like phase, with power-law distributed avalanches at any applied stress. This is in strong contrast to tuned criticality observed in depinninglike nonequilibrium phase transitions where criticality is observed only close to a critical point and is analogous to glassy systems where similar extended critical phases have been observed [25,33,34]. Thus, "extended criticality" seems to be a general feature of crystal plasticity of pure single crystals, irrespective of the spatial dimensionality of the system. Analogous ideas have very recently been presented also in the context of amorphous plasticity [35].

The final issue we address concerns the statistics of stress increments $\Delta \sigma$, i.e., the vertical segments in the top inset of Fig. 1(a); it is another quantity encoding information about the nature of the deformation process [36]. Figure 4 shows the $P_{\mathrm{INT}}(\Delta \sigma)$ distributions of all stress increments along the stress-strain curves separating strain bursts larger than $s^{\prime *}$. These are power-law distributed up to a $N_{0}$ dependent cutoff. The average stress increment $\langle\Delta \sigma\rangle$ decreases with $N_{0}$ as $\langle\Delta \sigma\rangle \propto N_{0}^{-0.5}$ (Fig. 4, inset). A similar power law dependence of stress increments on the system size is measured experimentally in molybdenum micropillars [12].

\section{SUMMARY}

To conclude, we have shown that bursty three-dimensional crystal plasticity cannot be envisaged in terms of a depinning transition but is rather a manifestation of an extended criticallike phase, reminiscent of glassy systems $[25,33,34]$. Interesting extensions of our study could be performed by adding a significant population of pinning centers, representing the effect of various additional defects such as precipitates [37], acting as obstacles for dislocation motion. Recent 2D studies [19] suggest that when in the competition between dislocation jamming and pinning due to obstacles the latter starts to dominate, a depinninglike scenario may be recovered. Our results point out the possibility that there are several universality classes in mesoscopic plasticity starting from the pure case studied here. Thus, the possible role of, e.g., the crystal structure (FCC vs BCC, etc. $[14,38]$ ) in determining the dislocation avalanche statistics in mesoscale 3D plasticity should be addressed. Our results await in-depth experimental studies.

\section{ACKNOWLEDGMENTS}

A.L., L.L., and M.J.A. are supported by the Academy of Finland through projects 13260053 and 251748 (Centres of Excellence Programme, 2012-2017) and acknowledge the computational resources provided by the Aalto University School of Science "Science-IT" project, as well as those provided by CSC (Finland). L.L. is supported by an Academy Research Fellowship (project no. 268302). S.Z. acknowledges support from the Academy of Finland FiDiPro program, project 13282993. G.C. and S.Z. are supported by the European Research Council Advanced Grant No. 291002 SIZEFFECTS.
[1] M. Zaiser, Scale invariance in plastic flow of crystalline solids, Adv. Phys. 55, 185 (2006).

[2] M. J. Alava, L. Laurson, and S. Zapperi, Crackling noise in plasticity, Eur. Phys. J. Special Topics 223, 2353 (2014).
[3] G. Ananthakrishna, Current theoretical approaches to collective behavior of dislocations, Phys. Rep. 440, 113 (2007).

[4] J. P. Sethna, K. A. Dahmen, and C. R. Myers, Crackling noise, Nature (London) 410, 242 (2001). 
[5] M. D. Uchic, P. A. Shade, and D. M. Dimiduk, Plasticity of micromater-scale single crystals in compression, Annu. Rev. Mater. Res. 39, 361 (2009).

[6] D. M. Dimiduk, C. Woodward, R. LeSar, and M. D. Uchic, Scale-free intermittent flow in crystal plasticity, Science 312, 1188 (2006).

[7] F. F. Csikor, C. Motz, D. Weygand, M. Zaiser, and S. Zapperi, Dislocation avalanches, strain bursts, and the problem of plastic forming at the micrometer scale, Science 318, 251 (2007).

[8] N. Friedman, A. T. Jennings, G. Tsekenis, J.-Y. Kim, M. Tao, J. T. Uhl, J. R. Greer, and K. A. Dahmen, Statistics of Dislocation Slip Avalanches in Nanosized Single Crystals Show Tuned Critical Behavior Predicted by a Simple Mean Field Model, Phys. Rev. Lett. 109, 095507 (2012).

[9] R. Maass, M. Wraith, J. T. Uhl, J. R. Greer, and K. A. Dahmen, Slip statistics of dislocation avalanches under different loading modes, Phys. Rev. E 91, 042403 (2015).

[10] K. S. Ng and A. H. W. Ngan, Stochastic nature of plasticity of aluminum micro-pillars, Acta Mater. 56, 1712 (2008).

[11] S. Brinckmann, J.-Y. Kim, and J. R. Greer, Fundamental Differences in Mechanical Behavior between Two Types of Crystals at the Nanoscale, Phys. Rev. Lett. 100, 155502 (2008).

[12] M. Zaiser, J. Schwerdtfeger, A. S. Schneider, C. P. Frick, B. G. Clark, P. A. Gruber, and E. Arzt, Strain bursts in plastically deforming molybdenum micro- and nanopillars, Philos. Mag. 88, 3861 (2008).

[13] S. Papanikolaou, D. M. Dimiduk, W. Choi, J. P. Sethna, M. D. Uchic, C. F. Woodward, and S. Zapperi, Quasi-periodic events in crystal plasticity and the self-organized avalanche oscillator, Nature (London) 490, 517 (2012).

[14] J. Weiss, W. B. Rhouma, T. Richeton, S. Dechanel, F. Louchet, and L. Truskinovsky, From Mild to Wild Fluctuations in Crystal Plasticity, Phys. Rev. Lett. 114, 105504 (2015).

[15] J. Weiss and J. R. Grasso, Acoustic emission in single crystals of ice, J. Phys. Chem. B 101, 6113 (1997).

[16] M.-C. Miguel, A. Vespignani, S. Zapperi, J. Weiss, and J.-R. Grasso, Intermittent dislocation flow in viscoplastic deformation, Nature (London) 410, 667 (2001).

[17] P. D. Ispánovity, L. Laurson, M. Zaiser, I. Groma, S. Zapperi, and M. J. Alava, Avalanches in 2D Dislocation Systems: Plastic Yielding Is Not Depinning, Phys. Rev. Lett. 112, 235501 (2014).

[18] S. Janicevic, M. Ovaska, M. J. Alava, and L. Laurson, Avalanches in 2D dislocation systems without applied stresses, J. Stat. Mech. (2015) P07016.

[19] M. Ovaska, L. Laurson, and M. J. Alava, Quenched pinning and collective dislocation dynamics, Sci. Rep. 5, 10580 (2015).

[20] O. Kapetanou, D. Weygand, and M. Zaiser, Stress and strain fluctuations in plastic deformation of crystals with disordered microstructure, J. Stat. Mech. (2015) P08009.

[21] C. Fressengeas, A. J. Beaudoin, D. Entemeyer, T. Lebedkina, M. Lebyodkin, and V. Taupin, Dislocation transport and intermittency in the plasticity of crystalline solids, Phys. Rev. B 79, 014108 (2009).

[22] A. Arsenlis, W. Cai, M. Tang, M. Rhee, T. Oppelstrup, G. Hommes, T. Pierce, and V. Bulatov, Enabling strain hardening simulations with dislocation dynamics, Model. Simul. Mater. Sci. Eng. 15, 553 (2007).

[23] B. Alessandro, C. Beatrice, G. Bertotti, and A. Montorsi, Domain-wall dynamics and Barkhausen effect in metallic ferromagnetic materials. I.Theory, J. Appl. Phys. 68, 2901 (1990).

[24] See Supplemental Material at http://link.aps.org/supplemental/ 10.1103/PhysRevB.94.064101 for results obtained using an alternative definition of the strain bursts, and a movie illustrating the nature of the bursty deformation process.

[25] B. Bakó, I. Groma, G. Györgyi, and G. T. Zimányi, Dislocation Glasses: Aging during Relaxation and Coarsening, Phys. Rev. Lett. 98, 075701 (2007).

[26] Vasily V. Bulatov, M. Rhee and W. Cai, Periodic boundary conditions for dislocation dynamics simulations in three dimensions, Mat. Res. Soc. Symp. 653, Z1.3.1 (2001).

[27] X. Zhang, B. Pan, and F. Shang, Scale-free behavior of displacement bursts: Lower limit and scaling exponent, Europhys. Lett. 100, 16005 (2012).

[28] L. Laurson, G. Durin, and S. Zapperi, Universality classes and crossover scaling of Barkhausen noise in thin films, Phys. Rev. B 89, 104402 (2014).

[29] J. Alcala, J. Ocenasek, K. Nowag, D. Esque-de los Ojos, R. Ghisleni, and J. Michler, Strain hardening and dislocation avalanches in micrometer-sized dimensions, Acta Mater. 91, 255 (2015).

[30] P. Bak, C. Tang, and K. Wiesenfeld, Self-Organized Criticality: An explanation of the $1 / f$ noise, Phys. Rev. Lett. 59, 381 (1987).

[31] G. Durin and S. Zapperi, The role of stationarity in magnetic crackling noise, J. Stat. Mech. (2006) P01002.

[32] J. Weiss, T. Richeton, F. Louchet, F. Chmelik, P. Dobron, D. Entemeyer, M. Lebyodkin, T. Lebedkina, C. Fressengeas, and R. J. McDonald, Evidence for universal intermittent crystal plasticity from acoustic emission and high-resolution extensometry experiments, Phys. Rev. B 76, 224110 (2007).

[33] M. Müller and M. Wyart, Marginal stability in structural, spin and electron glasses, Annu. Rev. Condens. Matter Phys. 6, 177 (2015).

[34] F. Pázmándi, G. Zaránd, and G. T. Zimányi, Self-Organized Criticality in the Hysteresis of the Sherrington-Kirkpatrick Model, Phys. Rev. Lett. 83, 1034 (1999).

[35] J. Lin, T. Gueudre, A. Rosso, and M. Wyart, Criticality in the Approach to Failure in Amorphous Solids, Phys. Rev. Lett. 115, 168001 (2015).

[36] J. Lin, E. Lerner, A. Rosso, and M. Wyart, Scaling description of the yielding transition in soft amorphous solids at zero temperature, PNAS 111, 14382 (2014).

[37] A. Lehtinen, F. Granberg, L. Laurson, K. Nordlund, and M. J. Alava, Multiscale modeling of dislocation-precipitate interactions in Fe: From molecular dynamics to discrete dislocations, Phys. Rev. E 93, 013309 (2016).

[38] P. Biscari, M. F. Urbano, A. Zanzottera, and G. Zanzotto, Intermittency in crystal plasticity informed by lattice symmetry, J. Elasticity 123, 85 (2015). 